\title{
Resistência ao impacto da madeira de nogueira-pecã em diferentes condições de umidade
}

\author{
Impact strength of nogueira-pecã wood on different moisture conditions
}

\author{
Rafael Beltrame $^{I^{*}}$ Bruno Dufau Mattos $^{\text {II }}$ Darci Alberto Gatto ${ }^{\mathrm{I}}$ Marília Lazarotto ${ }^{\mathrm{II}}$ \\ Clovis Roberto Haselein ${ }^{\text {IV }}$ Elio José Santini ${ }^{\mathrm{IV}}$
}

\section{RESUMO}

Os estudos de resistência da madeira ao impacto referem-se à eficiência com que este material absorve energia de um impacto e dissipa-a sem danos a sua estrutura. Dessa forma, o objetivo deste estudo foi avaliar a influência do teor de umidade na resistência ao impacto da madeira de nogueirapecã (Carya illinoinensis). Para tanto, foram utilizadas árvores procedentes de duas regiões fisiográficas do estado do Rio Grande do Sul. Os corpos de prova, em condições de equilíbrio a $12 \%$ de umidade e saturados, foram submetidos ao impacto utilizando-se pêndulo de CHARPY, e avaliados quanto à resistência oferecida com a aplicação da carga nos planos tangencial e radial e posições de retirada (medula - casca) nas toras, para as duas regiões fisiográficas em cada condição de umidade. Para auxiliar na interpretação dos dados, determinouse a massa específica aparente a $12 \%$ e saturada, trabalho absorvido, coeficiente de resiliência e a cota dinâmica. Pôde-se verificar, por meio dos resultados, que a madeira de nogueirapecã é mais resistente ao impacto na condição saturada.

Palavras-chave: flexão dinâmica, qualidade da madeira, madeira saturada, Carya illinoinensis.

\section{ABSTRACT}

The studies of wood impact strength refers to the efficiency of this material in absorb impact energy and dissipate it without structural damages. The present study aimed to evaluate the influence of moisture content on the impact strength of nogueira-pecã (Carya illinoinensis) wood. For this, trees from two physiographic regions of state of Rio Grande do Sul were used. The samples, stabilized at $12 \%$ of moisture content and in green conditions (saturated), were submitted to impact tests through a CHARPY pendulum, and were evaluated for the resistance to the application of loads in the tangential and radial sections, and in the positions of the log (pith-bark) for the two physiographic regions at each moisture condition. Moreover, the apparent specific gravity at $12 \%$ and in green conditions (saturated), the absorbed work, the resilience coefficient and the dynamic quote were determined. The main results showed that the nogueira-pecã wood is more resistant to the impact when it is in green conditions.

Key words: dynamic bending, wood quality, saturated wood, Carya illinoinensis.

\section{INTRODUÇÃo}

Por milhares de anos, diversas espécies florestais vêm sendo utilizadas para a produção de madeira e muitas delas têm sido utilizada pelo homem como um material resistente ao impacto, mas, surpreendentemente, pouco se sabe sobre a natureza exata de sua capacidade de absorção e dissipação de energia. Muitas dessas madeiras de elevada resistência apresentam uma estrutura de grã cruzada que determina a resistência ao rachar, quando carregada ao longo do sentido da grã. No entanto, além dessas observações, os mecanismos de absorção de energia e resistência ao impacto não são claramente entendidos (HEPWORTH, 2002).

A eficiente utilização de qualquer material, principalmente para fins estruturais, está condicionada,

'Centro de Engenharias, Universidade Federal de Pelotas (UFPel), Rua Conde Porto Alegre, 793, 96010-290, Pelotas, RS, Brasil.

E-mail: browbeltrame@yahoo.com.br. *Autor para correspondência.

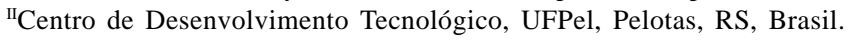

IIIDepartamento de Defesa Fitossanitária, Universidade Federal de Santa Maria (UFSM), Santa Maria, RS, Brasil.

${ }^{\text {IV }}$ Departamento de Ciências Florestais, UFSM, Santa Maria, RS, Brasil. 
entre outras características, ao conhecimento de suas propriedades físico-mecânicas, tanto no que se refere aos aspectos de segurança, quanto aos de economicidade. Dessa forma, a madeira merece especial destaque, em função da grande variabilidade que suas propriedades apresentam em comparação com as de outros materiais, da sua adequabilidade a inúmeras utilizações e da enorme variedade de espécies existentes (LISBOA et al., 1993).

Por outro lado, a madeira é um material heterogêneo, possuindo diferentes tipos de células, adaptadas ao desempenho de funções específicas e com grandes variações entre as espécies. Assim, vários estudos têm sido realizados visando a identificar quais os fatores que afetam as propriedades físico-mecânicas da madeira e os que podem ser inerentes ao próprio material ou ao ambiente onde as árvores se desenvolveram (LOBÃO et al., 2004).

Os avanços tecnológicos no setor industrial madeireiro têm permitido melhorar o aproveitamento da madeira e de seus derivados, criando novos produtos e ampliando sua aplicação. Dependendo do uso futuro de uma determinada peça de madeira, essa deverá ser capaz de suportar algumas condições adversas como uma sobrecarga ou eventual impacto.

Sabe-se que a flexão dinâmica de um material é definida como sendo a capacidade de determinada peça em resistir ao impacto. Essa propriedade mecânica tem importância na vida prática, especialmente quando utilizamos a madeira como cabos de ferramentas, mastros, na construção de escadas, carrocerias, aeronaves e determinados artigos esportivos (MORESCHI, 2005).

A resistência ao impacto de um corpo sólido depende diretamente de sua capacidade em absorver energia e dissipá-la por meio de deformações (MORESCHI, 2005) e alguns fatores podem influenciar nessa resistência, tais como, a forma e as dimensões dos corpos de prova, os ângulos das fibras, a massa específica, o teor de umidade, a temperatura e as propriedades anatômicas da madeira.

Um número grande de espécies lenhosas destaca-se como sendo particularmente resistente ao impacto em relação a sua massa específica, como, por exemplo, a nogueira (Carya sp.), enquanto outras espécies de massa específica semelhante, tais como o carvalho branco (Quercus alba), são muitos menos resistentes (HEPWORTH et al., 2002). Por sua vez, a madeira de nogueira-pecã (Carya illinoinensis (Wangenh) K. Koch), representante da família Juglandaceae, é bastante plantada no Estado do Rio
Grande do Sul com finalidades de produção de nozes comestíveis (GATTO et al., 2008). Apresenta um grande porte e tronco retilíneo, atingindo até $50 \mathrm{~m}$ de altura e diâmetro de até $150 \mathrm{~cm}$. Estudos realizados com o vergamento dessa madeira demonstram afinidade para a fabricação de móveis vergados (GATTO et al., 2006).

Este trabalho tem como objetivo determinar a resistência ao impacto da madeira de Carya illinoinensis (Wangenh) K. Koch (nogueira-pecã) sob duas condições de umidade.

\section{MATERIAL E MÉTODOS}

Para o estudo, foram amostradas pela extração, ao acaso, conforme norma da Comissión Panamericana de Normas Técnicas (COPANT, 1971), dez árvores adultas da espécie Carya illinoinensis, cinco provenientes da Encosta Superior do Nordeste do Rio Grande do Sul - Região 1 - e as outras cinco da Depressão Central - Região 2.

De cada árvore abatida, retirou-se uma tora de $3 m$ (Figura 1a), da qual se confeccionou um pranchão central, de 8cm de espessura com a medula incluída, e bem orientada (American Society for Testing and Materials-ASTM D-5536, 1995). Todo o material foi transportado para o Laboratório de Produtos Florestais do Departamento de Ciências Florestais da Universidade Federal de Santa Maria, onde os pranchões foram gradeados em local arejado para uma secagem lenta e gradual. Depois de secos, a espessura dos pranchões foi reduzida de 8,0 para $6,0 \mathrm{~cm}$ por meio de uma plaina desengrossadeira (Figura 1b). Em seguida, foram confeccionados oito corpos de prova, quatro próximos à casca (lenho adulto) e quatro próximos à medula (lenho juvenil), com dimensões de 2,0x2,0x28,0cm (Figura 1c), totalizando 80 corpos de prova para a realização dos ensaios.

Os 80 corpos de prova foram acondicionados em uma câmara climatizada nas condições de $20^{\circ} \mathrm{C}$ de temperatura e $65 \%$ de umidade relativa até atingirem umidade de equilíbrio em torno de $12 \%$. Após a climatização (30 dias), 40 corpos de prova, (20 lenho juvenil e 20 lenho adulto) foram submersos em água até sua saturação.

Os corpos de prova a $12 \%$ de umidade e saturados foram pesados em uma balança analítica de precisão 0,01g e, posteriormente, mensuradas suas dimensões com o uso de um paquímetro digital para determinação de seu volume (método estereométrico). Então, de posse desses dados, pode-se calcular a massa específica aparente correspondente ao teor de $12 \%$ de umidade e saturado (Equação 1), conforme DURLO(1991).

Ciência Rural, v.42, n.9, set, 2012. 


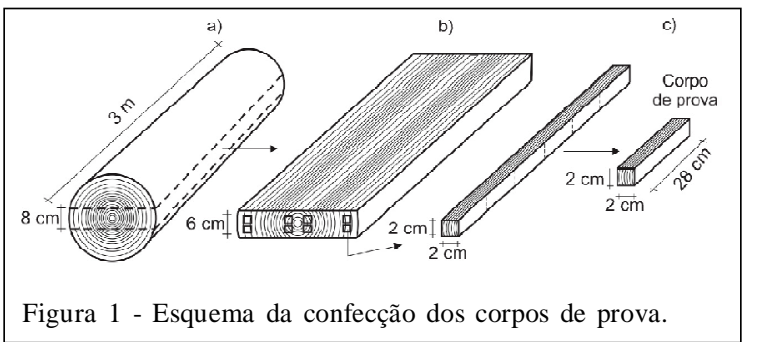

$$
\mathrm{ME}_{\mathrm{tu}}=\frac{\mathbf{M}_{\mathrm{tu}}}{\mathrm{V}_{\mathrm{tu}}}
$$

em que: $\mathrm{ME}_{\mathrm{tu}}=$ massa específica aparente ao teor de umidade (g. $\left.\mathrm{cm}^{-3}\right) ; \mathrm{M}_{\mathrm{tu}}=$ massa ao teor de umidade (g); $\mathrm{V}_{\mathrm{tu}}=$ volume ao teor de umidade $\left(\mathrm{cm}^{3}\right)$.

Para a realização dos testes de resistência ao impacto (flexão dinâmica), foi utilizado um pêndulo de CHARPY com capacidade de 100 joules. Os corpos de prova foram testados quanto aos sentidos da resistência ao impacto, radial e tangencial, e posições de retirada (próxima à medula e próxima à casca) das duas regiões fisiográficas para as condições de umidade a $12 \%$ e saturada.

Os corpos de prova foram colocados no vão da máquina de $24,0 \mathrm{~cm}$ e atingidos em sua região central pelo pêndulo. Após a queda do pêndulo, de uma altura de $1 \mathrm{~m}$, ocorre o impacto com o corpo de prova, obtendo-se o trabalho absorvido (W) em joules, constatado na leitura da escala graduada. Para fins de cálculo, transformou-se o trabalho absorvido de joule para kgm, usando a relação $1 \mathrm{~J}=0,102 \mathrm{kgm}$.

Obtidos os resultados do trabalho absorvido (W), calculou-se o coeficiente de resiliência (Equação 2) para fins práticos, segundo a L'A Association Francaise de Normalization (AFN, 1942).

$$
\mathrm{K}=\frac{\mathrm{W}}{\mathrm{b}(\mathrm{h}){ }^{10} / 6}
$$

em que: $\mathrm{K}$ = coeficiente de resiliência $\left(\mathrm{kgm} . \mathrm{cm}^{-2}\right) ; \mathrm{W}=$ trabalho absorvido para romper o corpo de prova (kgm); $\mathrm{b}$ e $\mathrm{h}=$ dimensões transversais do corpo de prova $(\mathrm{cm})$.
A cota dinâmica (Equação 3) é outro valor a ser calculado, com a finalidade de comparar diferentes madeiras, mas com correção para que a influência causada pela variação em massa específica entre espécies seja eliminada (MORESCHI, 2005).

$$
\mathrm{CD}=\frac{\mathrm{K}}{\mathrm{D}^{2}}
$$

em que: $C D$ = cota dinâmica; $K=$ coeficiente de resiliência $\left(\mathrm{kgm} . \mathrm{cm}^{-2}\right)$; $\mathrm{D}=$ massa específica aparente (g.cm ${ }^{-3}$ ).

Posterior à realização dos testes de flexão dinâmica, amostras laterais de 5,0cm retiradas dos corpos de prova na condição a $12 \%$ de umidade e saturados foram pesadas e colocadas em estufa a $103^{\circ} \mathrm{C}$ até atingirem massa constante. De posse desses valores, calculou-se o teor de umidade da madeira através da Equação 4:

$$
\mathrm{TU}_{\mathbf{t}}=\frac{\mathbf{M}_{\mathrm{t}}-\mathbf{M}_{0}}{\mathbf{M}_{0}} * 100
$$

em que: Tut $=$ Teor de umidade testado (\%); Mut = Massa do corpo de prova testado em cada condição de umidade (g); Mo = Massa seca a $0 \%$ de umidade (g).

Para o estudo do comportamento da flexão dinâmica, os dados foram submetidos à análise de variância com posterior comparação por teste de médias pelo teste de Tukey, a 5\% de probabilidade de erro e análise de regressão, utilizando o pacote estatístico Statgraphics Plus e adotando 5\% de probabilidade de erro.

\section{RESULTADOS E DISCUSSÃO}

As variáveis apresentaram maiores médias para a madeira na condição saturada (Tabela 1). Os valores de $\mathrm{W}$ e $\mathrm{K}$ foram aproximadamente 2,5 e 2 vezes maiores para a madeira na condição saturada em relação à madeira a $12 \%$ de umidade e essa tendência de aumento da resistência com o acréscimo da umidade corrobora o verificado anteriormente por KRECH(1960) eBELTRAME (2010).

Tabela 1 - Valores médios dos parâmetros de resistência ao impacto da madeira para as duas condições de umidade.

\begin{tabular}{lcccc}
\hline Condição & MEA $\left(\mathrm{g} \mathrm{cm}^{-3}\right)$ & W $(\mathrm{Kgm})$ & $\mathrm{K}(\mathrm{Kgm} \mathrm{cm})^{-2}$ & $\mathrm{CD}$ \\
\hline $12 \%$ umidade & $0,814 \pm 0,05 \mathrm{~A}$ & $2,976 \pm 0,95$ A & $0,449 \pm 0,14$ A & $0,680 \pm 0,22 A$ \\
Saturada & $1,160 \pm 0,14 B$ & $7,774 \pm 2,37$ B & $0,942 \pm 0,28$ B & $0,704 \pm 0,22 A$ \\
\hline
\end{tabular}

Em que: Média $a_{ \pm D}$. Médias não seguidas pela mesma letra na coluna possuem diferença estatisticamente significativa entre si em nível de 5\% de significância, de acordo com o Teste HSD de Tukey. 
Os resultados evidenciam que a madeira dessa espécie torna-se mais elástica com o acréscimo de umidade e é capaz de absorver e dissipar melhor as cargas de choque a ela imposta. PEDROSO \& MATOS (1987), ao estudarem madeiras de Eucalyptus sp. provenientes de plantios do Estado do Rio Grande do Sul, verificaram que a madeira a $12 \%$ de umidade, apresentou valores médios para o trabalho absorvido (W) de 1,6 a 7,2kgm, coeficiente de resiliência (K) entre 0,25 e 1,17 kgm.cm ${ }^{-2}$ e valores para cota dinâmica (CD) de 0,32 a 1,23. De tal modo, os valores encontrados neste estudo, para a condição de equilíbrio a 12 \% de umidade, condizem com estudos anteriormente realizados.

Os valores de W, K e CD encontrados neste estudo, para as duas condições de umidade, foram superiores aos encontrados anteriormente por BELTRAME et al. (2010) para a madeira de açoitacavalo. Nesse mesmo sentido, verificaram-se valores médios para $12 \%$ de umidade desse trabalho, superiores aos anteriormente encontrados por STANGERLIN et al. (2008) para a espécie de Eucalyptus saligna, para massa específica aparente (MEA) a 12\% de umidade muito próxima.

Ao considerar a similaridade entre a MEA a $12 \%$ da madeira de nogueira-pecã com a da madeira de Eucalyptus saligna, estudada por STANGERLIN et al. (2008), atribuiu-se a diferença de valores de flexão dinâmica à diferença na estrutura anatômica das espécies. HEPWORTH et al. (2002) afirmam que tais propriedades são grandes influenciadoras na capacidade da madeira em absorver cargas de choque e dissipá-las sem danos à sua estrutura.

Apesar de se ter comprovado o aumento das propriedades de resistência ao impacto, causado pelo alto teor de umidade da madeira, CHISTIANSEN
(1997), em seus estudos, comprovou que essas variações de teor de umidade na madeira podem gerar influências significativas nas propriedades de resistência mecânica, todavia, concluiu que essas variações não podem ser atribuídas completamente a essas modificações nos teores de umidade.

A região, a posição (medula e casca) e o sentido (tangencial e radial) não foram fatores relevantes nas variações de MEA, $\mathrm{W}$ e $\mathrm{K}$ nas duas condições de umidade (Tabela 2). Por outro lado, a cota dinâmica (CD) da madeira saturada foi influenciada pela região, apresentando superioridade estatisticamente significativa dos valores observados na região da Encosta Superior do Nordeste, em relação àqueles observados na Região da Depressão Central.

Pode-se afirmar que, para a madeira da espécie de nogueira-pecã, o W, K e a CD não podem ser estimados por meio da massa específica aparente, de acordo com os resultados observados na tabela 3. Essa mesma situação ocorreu anteriormente em estudos de STANGERLIN et al. (2008) para as espécies de Eucalyptus botrioides e Eucalyptus saligna e em relatos de BELTRAME et al. (2010), que não conseguiram bons ajustes para a madeira da espécie Lueha divaricata para essas propriedades. Tanto nesse como nos estudos anteriores, foram observados baixos coeficientes de determinação, assim como valores de probabilidades não significativos.

\section{CONCLUSÃO}

Em todas as variáveis referentes à resistência ao impacto da madeira de nogueira-pecã, ela apresentou melhores resultados para a condição saturada, quando comparada à condição de $12 \%$ de umidade.

Tabela 2 - Valores médios de massa específica aparente (MEA), trabalho absorvido (W), coeficiente de resiliência (K) e cota dinâmica (CD) para as diferentes Regiões, posições e sentidos da madeira de nogueira-pecã nas condições saturada e a 12\% de umidade.

\begin{tabular}{|c|c|c|c|c|c|c|c|c|}
\hline \multirow{2}{*}{ Variáveis } & \multicolumn{2}{|c|}{-----MEA $\left(\mathrm{g} \mathrm{cm}^{-3}\right)$------ } & \multicolumn{2}{|c|}{----'W (Kgm)------'- } & \multicolumn{2}{|c|}{----K $\left(\mathrm{Kgm} \mathrm{cm}^{-2}\right)$------ } & \multicolumn{2}{|c|}{-------------CD------------- } \\
\hline & $12 \%$ & Saturada & $12 \%$ & Saturada & $12 \%$ & Saturada & $12 \%$ & Saturada \\
\hline Região (1) & $0,804 a$ & $1,041 \mathrm{a}$ & $2,824 a$ & $5,503 a$ & $0,424 a$ & $0,695 a$ & $0,656 a$ & $0,627 \mathrm{a}$ \\
\hline Região (2) & $0,814 a$ & $1,024 a$ & $3,166 a$ & $6,545 a$ & $0,480 \mathrm{a}$ & $0,831 \mathrm{a}$ & $0,729 a$ & $0,772 b$ \\
\hline Medula & $0,825 a$ & $1,043 a$ & $2,968 a$ & $6,032 a$ & $0,444 a$ & $0,740 \mathrm{a}$ & $0,658 a$ & $0,673 a$ \\
\hline Casca & $0,798 a$ & $1,024 a$ & $3,029 a$ & 6,033a & $0,461 \mathrm{a}$ & $0,786 a$ & $0,722 \mathrm{a}$ & $0,727 a$ \\
\hline Tangencial & $0,820 \mathrm{a}$ & $1,069 \mathrm{a}$ & 2,919a & 6,558a & $0,437 a$ & $0,826 a$ & $0,643 a$ & $0,704 a$ \\
\hline Radial & $0,803 a$ & $1,004 a$ & $3,054 a$ & $5,612 \mathrm{a}$ & $0,463 a$ & $0,715 a$ & $0,722 \mathrm{a}$ & 0,698a \\
\hline
\end{tabular}

Em que: médias não seguidas por letras iguais sobrescritas na coluna possuem diferença estatisticamente significativa entre si em nível de 5\% de significância, de acordo teste HSD de Tukey; Região 1 = Depressão Central; Região 2 = Encosta Superior do Nordeste. 
Tabela 3 - Modelos de regressão para a estimativa do trabalho absorvido (W), coeficiente de resiliência (K) e cota dinâmica (CD) em razão da massa específica aparente (MEA) nas condições de 12\% de umidade e saturada

\begin{tabular}{|c|c|c|c|c|c|}
\hline Umidade & Equação de regressão & $\mathrm{R}_{\mathrm{aj}}{ }^{2}$ & $\mathrm{~S}_{\mathrm{yx}}$ & Razão F & Valor $\mathrm{P}$ \\
\hline \multirow{3}{*}{$12 \%$} & $\mathrm{~W}=0,846576+2,65966 * \mathrm{MEA}$ & 2,4 & 0,95 & 0,52 & 0,4800 \\
\hline & $\mathrm{K}=0,144625+0,381179 * \mathrm{MEA}$ & 2,1 & 0,15 & 0,44 & 0,5136 \\
\hline & $\mathrm{CD}=1,46182-0,946744 * \mathrm{MEA}$ & 5,2 & 0,23 & 1,15 & 0,2949 \\
\hline \multirow{3}{*}{ Saturada } & $\mathrm{W}=16,1882-7,24976 * \mathrm{MEA}$ & 2,0 & 1,82 & 0,79 & 0,3786 \\
\hline & $\mathrm{K}=1,19927-0,220863 * \mathrm{MEA}$ & 0,2 & 0,22 & 0,05 & 0,8231 \\
\hline & $\mathrm{CD}=2,52562-1,56897 * \mathrm{MEA}$ & 10,3 & 0,16 & 4,35 & 0,0437 \\
\hline
\end{tabular}

Em que: $\mathrm{R}_{\mathrm{aj}}{ }^{2}=$ coeficiente de determinação ajustado (\%); $\mathrm{S}_{\mathrm{yx}}=$ erro padrão de estimativa; Razão $\mathrm{F}=$ valor de $\mathrm{F}$ calculado; Valor $\mathrm{P}=$ nível de probabilidade de erro.

\section{REFERÊNCIAS}

AMERICAN SOCIETY for Testing and Materials. ASTM D5536-94: Standard methods of testing small clear specimens of timber. Philadelphia, 1995. 9p.

BELTRAME, R. et al. Resistência ao impacto da madeira de açoita-cavalo em diferentes condições de umidade. Cerne Lavras v.16, n.4, 2010. Disponível em: <http://www.dcf.ufla.br/ cerne/artigos/03-02-20115854v16_n4_artigo\%2009.pdf $>$. Acesso em: 19 abr. 2012

CHRISTIANSEN, A.W. Effect of overdrying on toughness of yellowpoplar veneer. Holz al Roh-und Werkstoff (European Journal of Wood and Wood Products), Springer- Verlag v.55, p.71-75, 1997. Disponível: <http://www.fpl.fs.fed.us/ documnts/pdf1997/chris97a.pdf>. Acesso em: 19 abr. 2012.

COMISSIÓN PANAMERICANA DE NORMAS TÉCNICAS COPANT 30:1 - 001: Selección y colección de maderas. Caracas, 1971. 5p.

DURLO, M.A. Tecnologia da madeira: peso específico. Santa Maria: UFSM, CEPEF/ FATEC, 1991. 29p. (Série Técnica, n.8).

GATTO, D.A. Características tecnológicas do vergamento das madeiras de Luehea divaricata, Carya illinoinensis e Platanus $x$ acerifolia como subsídio para o manejo florestal. 2006. 115f. Tese (Doutorado em Engenharia Florestal) Curso de Pós-graduação em Engenharia Florestal, Universidade Federal de Santa Maria, RS.

GATTO, D.A. et al. Características tecnológicas das madeiras de Luehea divaricata, Carya illinoinensis e Platanus x acerifolia quando submetidas ao vergamento. Ciência Florestal, Santa Maria, v.18, n.1, p.121-131, 2008. Disponível em: <http:// www.ufsm.br/cienciaflorestal/artigos/v18n1/A11V18N1.pdf>. Acesso em: 19 abr. 2012.

HEPWORTH, D. G. et al. Variations in the morphology of wood structure can explain why hardwood species of similar density have very different resistances to impact and compressive loading. Philosophical Transactions Royal Society London. Londres, v.360, p.255-272, 2002. Disponível em: <http://rsta.royalsocietypublishing.org/content/360/1791/ 255.full.pdf $>$. Acesso em: 05 jan. 2011. doi: 10.1098/ rsta.2001.0927.

KRECH, H. Größe und zeitlicher Ablauf von Kraft und Durchbiegung beim Schlagbiegeversuch Holz und ihr Zusammenhang mit der Bruchschlagarbeit. SpringerVerlag, 1960. 11p.

L'A Association Francaise de Normalization - AFN. NF B51009: Norme Francaise, bois essai de choc ou flexion dynamique. Paris, 1942. 3p.

LISBOA, C.D.J. et al. Amostragem e propriedades físicomecânicas de madeiras amazônicas. Brasília: Ministério do Meio Ambiente e da Amazônia Legal IBAMA, 1993. 103p. (Coleção Meio Ambiente. Série Estudos Floresta, 1).

LOBÃO, M.S. et al. Caracterização das propriedades físicomecânicas da madeira de eucalipto com diferentes densidades. Revista Árvore, Viçosa, v.28, n.6, p.889-894, 2004. Disponível em: <http://www.scielo.br/pdf/rarv/v28n6/ 23990.pdf>. Acessado em: 12 dez. 2010.

MORESCHI, J.C. Propriedades tecnológicas da madeira Manual Didático. Curitiba: UFPR, 2005. 124p.

PEDROSO, O.; MATTOS, J.R. Estudo sobre madeiras do Rio Grande do Sul. Porto Alegre: Instituto de Pesquisas de Recursos Naturais Ataliba Paz, 1987. 181p. (Publicação IPRNR, n.20).

STANGERLIN, D.M. et al. Avaliação da resistência ao impacto para as madeiras de Eucalyptus botrioides e Eucalyptus saligna. In: CONGRESSO FLORESTAL ESTADUAL, 10., 2008, Nova Prata, RS. Anais... Nova Prata: Congresso Florestal Estadual, 10., 2009. 1 CD, 154p. p.65. 